Editorial

\title{
Computational Topology in Image Context
}

CTIC - Computational Topology in Image Context - is a series of annual international workshops devoted to gather researchers dealing with the study of topological invariants from the computational point of view, and/or who want to use topological information in image applications. The workshops intend to provide an opportunity for participants, from different fields related to computational geometry, discrete geometry, geometrical modeling, algebraic topology and image processing, to exchange ideas.

CTIC has been held in Poitiers, France (2008) and St Catherine, Austria (2009). The third edition of this series was held in Chipiona, Spain, 10-12 November 2010. The next CTIC workshop will be held in Bertinoro, Italy, 28-30 May 2012. Initially, this workshop series pretended to act as a catalyst for creating new collaborations, and starting new interdisciplinary projects. To achieve this goal, participants were encouraged to present an up-to-date survey of their research related to one of the topics of the workshop (which include computational geometry, discrete geometry, geometrical modeling, algebraic topology and image processing). Recent but yet unpublished results, reports on research in progress, and recently published results (only considered for oral presentations) were submitted. Works related to image applications using topological invariants were particularly welcome.

26 Top-quality scientific papers were accepted to be presented at CTIC2010, based on the scientific reviews from 31 papers submitted. Two internationally recognized speakers accepted our invitation to present stimulating research talks: Claudia Landi, Bologna University (Italy) and Herbert Edelsbrunner, Duke University (USA). 14 Papers were selected to be extended for this special issue. After the reviewing process, 9 of them were accepted.

The first paper entitled "A Point Calculus for Interlevel Set Homology" describes a recent development to extend persistence diagram which is a multiset of points in the extended plane representing the set of all persistent homology groups of a given real-valued tame function (a continuous function with only finitely many critical values and such that every lower level set has only finite rank homology groups). It is used to compute homology, and maps between homology, of all possible combinations of interlevel sets and relative pairs of interlevel sets of real-valued functions. The paper is written with an eye on minimizing the formalism and adding intuitive explanations where the formalism seemed necessary or useful.

The second paper, "Persistent Homology and Partial Similarity of Shapes", also deals with the theory of Persistent Homology. In this paper, persistence diagrams are used to address the problem of shape comparison based on partial similarity. In general, two shapes having a common sub-part present a common persistent sub-diagram. Hence, the partial Hausdorff distance between persistence diagrams measures partial similarity between shapes.

The third paper, entitled "How to Define a Locally Adaptive Sampling Criterion for Topologically Correct Reconstruction of Multiple Regions", specifies locally adaptive sampling criteria for reconstructing non-manifold surfaces in a topologically correct way.

The fourth paper presents a novel algorithm to optimize the reconstruction from non-uniform point sets. This approach offers a valuable method for the evaluation of the reconstruction process without the need of visual inspection of the reconstructed database.

In the fifth paper, a fast algorithm to compute cohomology groups generators of cellular decomposition of any orientable 2-manifold is described.

The sixth paper, entitled "Ronse deletability conditions and (N,k)-retractions", provides a link between digital retractions and parallel thinning algorithms. More concretely, it gives a full characterization of the thinning algorithms that can be modeled as ( $\mathrm{N}, \mathrm{k}$ )-retractions (a particular kind of multivalued retraction with the property that each point is retracted to its neighbors).

A definite theory of discrete surfaces is still an open problem in Digital Topology. In the seventh paper, a new family of discrete surfaces in the grid Z3 which are based on the use of certain discrete patterns (plates) in the role of polygonal faces is given.

The last two papers deal with the problem of obtaining discrete Morse functions on cell complexes. The first paper entitled "Perfect Discrete Morse functions on 2-complexes" focuses on the study of discrete Morse functions such that the number of critical simplicies coincides with the Betti number of the complex in each dimension, on a 2D simplicial complex. The second paper introduces the foundations of a homology-based heuristic for finding optimal discrete gradient vector fields (or equivalently, optimal discrete Morse functions) on a general finite cell complex.

We like to thank Gabriella Sanniti di Baja, Editor-in-Chief of Pattern Recognition Letter, for giving us the opportunity to edit this special issue and Anjana Jayaram, Journal Manager, from the editorial office, for their support in managing the issue. Our thanks go to the reviewers for their valuable comments to improve the quality of the papers. 